\title{
Dynamic path controllability in economic models
}

\author{
From linearity to nonlinearity*
}

\author{
W.C.A. Maas and H. Nijmeijer \\ University of Twente, 7500 AE Enschede, The Netherlands
}

Received July 1992, final version received March 1993

In this paper we study the dynamic path controllability of discrete-time nonlinear economic models in state-space form. First we present an algorithmic procedure for testing this property for time-varying linear systems. Secondly we extend this method to general nonlinear models and we show that under generic conditions path controllability around a specific trajectory of the nonlinear system is equivalent to path controllability of the corresponding linearized model. The results are illustrated with two economic examples.

Key words: State-space model; Discrete time; Dynamic path controllability; Linearization JEL classification: $\mathrm{C} 67 ; \mathrm{O} 21 ; \mathrm{C} 30$

\section{Introduction}

In macroeconomic theory a standard question dcals with the ability of guiding a given set of target trajectories (as functions of time) along desired paths through an appropriate choice of instrument variables at each time instant. 'This is usually refered to as the question of dynamic path controllability or perfect controllability. In case a positive answer to the above problem exists, the policy makers can steer the target variables along any given desired paths. One may view this as a dynamic interpretation of the well-known Tinbergen concept of static controllability; cf. Tinbergen (1952). In the study of the dynamic path controllability it is essential to have an exact description of the macroeconomic model under consideration. Of course, without knowledge about the

Correspondence to: H. Nijmeijer, Department of Applied Mathematics, University of Twente, P.O. Box 217, 7500 AE Enschede, The Netherlands. Fax: + 31-53-340733.

*The authors are grateful to the anonymous referees for three valuable comments on an earlier draft of this paper. 
dynamic structure of the system, it seems not reasonable to look for an adequate description of its path controllability. Thereforc, dynamic path controllability has been studied for a nice set of dynamic economic models in the economics literature by various authors, e.g., Aoki (1974, 1975, 1976), Buiter (1979), Aoki and Canzoneri (1979), Preston and Pagan (1982), Wohltmann (1984, 1985b), Wohltmann and Krömer (1983, 1984). In all these references the considered economic models are assumed to be linear and in state-space form. To our best knowledge there exists only a few results on perfect controllability for nonlinear economic models; see, e.g., Engwerda (1988a,b) where some partial results for time-varying linear systems are derived and Nijmeijer $(1989,1990 \mathrm{a}, \mathrm{b})$ for general nonlinear models. There also exists quite some engineering literature on dynamic path controllability, in that context usually referred to as rightinvertibility or functional reproducibility; see, e.g., Brockett and Mesarovic (1965), Sain and Massey (1969), and Wolovich (1974) for linear results and Albrecht, Grasse, and Wax (1981), Fliess (1986), Respondek and Nijmeijer (1988), and Respondek (1990) for nonlinear results.

As noted before, very few results on path controllability of nonlinear economic models are known. A standard approach to avoid nonlinearities is therefore to linearize the economic model about a fixed equilibrium state and then to apply the usual linear theory on dynamic path controllability; see, for instance, the examples of Wohltmann and Krömer (1984). That such an approach indeed may be justified under some additional generic assumptions has been shown in Nijmeijer (1989). Here generic is a mathematical phrasing for almost always. Unfortunately such results are of no use in situations where there is no natural equilibrium state; for instance this will occur in a dynamic economic model where some (time-varying) exogenous variables are present; see, for example, Chow (1977). In that case the linearization of the nonlinear economic model has to be performed around some relevant (state) trajectory. Obviously the resulting linearized model will also be time-varying, and so no definite results on the perfect controllability of the resulting time-varying linear model are available. The purpose of the present paper is twofold. Firstly, we will derive a necessary and sufficient condition for (uniform) dynamic path controllability of a time-varying linear system. Secondly, we will relate, in analogy to Nijmeijer (1989), the dynamic path controllability of a nonlinear economic model around a given state trajectory with that of the corresponding linear time-varying model. As we will establish in the sequel, we may conclude that under generic conditions the perfect controllability of the true nonlinear model is satisfied if and only if its time-varying linear model is. Since this latter statement is far more easy to verify, we believe that our paper contributes to the complete understanding of nonlinear economic models.

Throughout this work we consider discrete-time economic models. Mutatis mutandis, all our results seem to remain valid for continuous-time systems, but for conceptual reasons we are restricting ourselves to discrete-time results. For 
a detailed analysis in continuous-time dynamic path controllability we refer to the linear results of Wohltmann (1985a, b), and some partial nonlinear results are contained in Albrecht, Grasse, and Wax (1981), Respondek and Nijmeijer (1988), and Respondek (1990).

The paper is organized as follows. In section 2 we give the main definitions used in the paper, especially uniform path controllability and local dynamic path controllability, and formulate the problem we shall discuss. We shortly look at linear time-varying systems in section 3 . In this section we give a rank condition and an algorithm which can both be used to verify the uniform path controllability. In the following section we extend the algorithm mentioned in section 3 to check the path controllability for nonlinear models, and in section 4 we will come up with the main result of this note, namely the connection between the local dynamic path controllability of a nonlinear system and the uniform path controllability of its linearization around a certain time path. Two examples explaining and supporting the theory will be given in section 5 , after which we finish with some concluding remarks in section 6 .

\section{Definitions and problem formulation}

Throughout this note we consider economic models described in discrete-time and given by a state-space model of the form

$$
\begin{aligned}
q(t+1) & =f(q(t), u(t), w(t)), \\
y(t) & =h(q(t), u(t), w(t)), \quad t=0,1,2, \ldots,
\end{aligned}
$$

where $q$ is an $n$-dimensional state vector, $u$ is an $m$-dimensional instrument vector consisting of the policy variables that can be freely assigned, $w$ is an $r$-dimensional vector of known exogenous variables (the remaining policy variables, the policy variables from the external world acting on our economy, etc.), and $y$ is an $p$-dimensional vector of target variables that we want to control. The state transition map $f$, that describes the state evolution from a given state $q$, instrument $u$, and exogenous variable $w$ at a given time instant $t$, is supposed to he analytic I Ikewise the output map $h$ is supposed to be analytic (or at least sufficiently many times differentiable, where in this context many should be at least $n$, the dimension of the state). For convenience we consider model (1) if necessary initialized at $t=0$; since the system is time-invariant (i.e., not explicitly depending on time) this may be done without loss of generality. Often we consider a specific trajectory of (1), that is some specific set of time functions $(\bar{q}(t), \bar{u}(t), \bar{w}(t), \bar{y}(t))$ that satisfy these equations. Usually such a set may be obtained by specifying an initial state $\vec{q}(0)=\bar{q}_{0}$ and describing some instrument function $\bar{u}(t), t=0,1,2, \ldots$, and some exogenous variable $\bar{w}(t), t=$ $0,1,2, \ldots$, which via eq. (1) yield the state $\bar{q}(t), t=0,1,2, \ldots$, and output $\bar{y}(t)$, 
$t=0,1,2, \ldots$ Around a given trajectory $(\bar{q}(t), \bar{u}(t), \bar{w}(t), \bar{y}(t))$ of $(1)$ we consider the linearization or the first-order variation of (1) about $(\bar{q}(t), \bar{u}(t), \bar{w}(t), \bar{y}(t))$,

$$
\begin{aligned}
\tilde{q}(t+1) & =A(t) \tilde{q}(t)+B(t) \tilde{u}(t)+E(t) \tilde{w}(t), \\
\tilde{y}(t) & =C(t) \tilde{q}(t)+D(t) \tilde{u}(t)+F(t) \tilde{w}(t), \quad t=0,1,2, \ldots,
\end{aligned}
$$

where

$$
\begin{aligned}
& A(t)=\left(\frac{\partial f_{i}}{\partial q_{j}}(\bar{q}(t), \bar{u}(t), \bar{w}(t))\right)_{i, j=1, \ldots, n}, \\
& B(t)=\left(\frac{\partial f_{i}}{\partial u_{j}}(\bar{q}(t), \bar{u}(t), \bar{w}(t))\right)_{i=1, \ldots, n, j=1, \ldots, m} \\
& E(t)=\left(\frac{\partial f_{i}}{\partial w_{j}}(\bar{q}(t), \bar{u}(t), \bar{w}(t))\right)_{i=1, \ldots, n, j=1, \ldots, r} \\
& C(t)=\left(\frac{\partial h_{i}}{\partial q_{j}}(\bar{q}(t), \bar{u}(t), \bar{w}(t))\right)_{i=1, \ldots, p, j=1, \ldots, n} \\
& D(t)=\left(\frac{\partial h_{i}}{\partial u_{j}}(\bar{q}(t), \bar{u}(t), \bar{w}(t))\right)_{i=1, \ldots, p, j=1, \ldots, m} \\
& F(t)=\left(\frac{\partial h_{i}}{\partial w_{j}}(\bar{q}(t), \bar{u}(t), \bar{w}(t))\right)_{i=1, \ldots, p, j=1, \ldots, r}
\end{aligned}
$$

For simplicity we restrict ourself to systems of the above form in which the matrices $D(t), E(t)$, and $F(t)$ are equal to zero for all $t$. Mutatis mutandis, the results also hold for systems in which these matrices are not equal to zero. As stated in the introduction we are interested in path controllability. So we are looking under which conditions it is possible to steer the target variables $y$ of a specific model along any desired time path by a proper choice of the time path of the policy variables $u$.

For static linear models Tinbergen (1952) has proved that a necessary condition for path controllability is that the number of policy variables must be larger or equal to the number of target variables (the so-called Tinbergen condition).

In the following we assume that the number of target variables equals the number of policy variables. So

$$
p=m \text {. }
$$

The results, mutatis mutandis, also hold for models in which there are more policy variables than target variables. 
Given these simplifications, our model (1) will be of the following form:

$$
\begin{aligned}
q(t+1) & =f(q(t), u(t)), \\
y(t) & =h(q(t)), \quad t=0,1,2, \ldots .
\end{aligned}
$$

Its corresponding linearisation around a specific trajectory $(\bar{u}(t), \bar{q}(t), \bar{y}(t))$ will be

$$
\begin{aligned}
\tilde{q}(t+1) & =A(t) \tilde{q}(t)+B(t) \tilde{u}(t), \\
\tilde{y}(t) & =C(t) \tilde{q}(t),
\end{aligned}
$$

Now we will define path controllability for models (4) and (5) more properly.

We will call model (4) local dynamic path controllable around the given trajectory if there exist a lead $\alpha$, a lag $l$, and an $\varepsilon(>0)$ such that for every m-dimensional path $\xi(\cdot)$ with $\|\xi(t)-\bar{y}(t)\|<\varepsilon$ for all $\alpha+l-1 \geq t \geq \alpha$ it follows that there exists policy variables $u(t)$ for $\alpha+l-2 \geq t \geq 0$ such that the resulting target variables $y(t)$ will be equal to the path $\xi(t)$ for all $\alpha+l-1 \geq t \geq \alpha$.

So in a neighbourhood of the given trajectory $\bar{y}(\cdot)$ of (4) it is then possible to steer every target time path by a proper choice of the policy variables $u(t)$, $t=0,1,2, \ldots$

For linear model (5) we do not need to restrict ourselves to local dynamic path controllability. The linear model (5) will be called uniform path controllable if there exist a lead $\alpha$, a lag $l$ such that for every $m$-dimensional time path $\xi(\cdot)$ there exists policy variables $\tilde{u}(t)$ for $\alpha+l-2 \geq t \geq 0$ such that the resulting target variables $\tilde{y}(t)$ will be equal to $\xi(t)$ for $\alpha+l-1 \geq t \geq \alpha$. Uniform (target) path controllability as defined here is the same as target path controllability $\operatorname{TPC}(0 ; \alpha, l)$ as has been used in Engwerda (1988a, b), but we prefer to use the above terminology since it is consistent with existing literature on nonlinear discrete-time systems; $\mathrm{cf}$. Nijmeijer and van der Schaft (1990) and Nijmeijer (1990a, b).

The main problem we will discuss is the interesting question whether there is a link between the local dynamic path controllability of (4) around the trajectory $(\bar{u}(t), \bar{q}(t), \bar{y}(t))$ and the uniform path controllability of its linearization around this trajectory (5). As a particular case this includes the problem treated in Nijmeijer (1989), where the given target trajectory $\bar{y}(t), t=0,1,2, \ldots$, corresponds to a constant sequence since $\bar{y}(t)=\bar{y}$ is given as $\bar{y}=h(\bar{x})$, with $\bar{x}=$ $f(\bar{x}, \bar{u})$ an equilibrium of (4).

\section{Time-varying linear path controllability}

The key idea underlying the subsequent developments is that, if the timevarying matrices $A(\cdot), B(\cdot)$, and $C(\cdot)$ in (5) smoothly depend upon $t$, then we may 
introduce a particular nonlinear discrete-time system by defining as extended state vector $q=\left(\tilde{q}, q_{n+1}\right)$, where the last component simply denotes time. With this trick (5) is equivalent to an autonomous (not time-varying) system, given by the dynamic equation of (5) and, in addition, the extra equation $q_{n+1}(t+1)$ $=q_{n+1}(t)+1$. The autonomous system is obviously initialized at $\left(\tilde{q}_{0}, 0\right)$. In this way the linear time-varying system (5) may be studied along the lines of Nijmeijer and van der Schaft (1990) and Nijmeijer (1987, 1990b). In particular, we may study uniform target path controllability (also called right-invertibility) as in these references, which will be done in detail in the sequel. At this point it is useful to note that for (local) target path controllability of a nonlinear discretetime system the requirement that $m \geq p$ is necessary; cf. Nijmeijer and van der Schaft (1990) and Nijmeijer (1987, 1990b). This justifies the assumption that the number of target variables equals the number of policy variables; see (3) and the remark following (3).

In order to check the path controllability of the time-varying linear system (5) we apply the following algorithm to (5). This algorithm, in fact, constructs a dynamic mechanism (in engineering called a compensator) which decouples the model. So, after using this compensator the component $\tilde{y}_{i}$ of $\tilde{y}$ is only affected by $\tilde{u}_{i}$ and not by the other components of $\tilde{u}$, and as an easy inspection shows, the resulting model, i.e., the system together with the compensator, is dynamic path controllable [see Nijmeijer (1989) where the equivalence between decoupling and target path controllability is given]. Moreover, a crucial point in using the algorithm is that it directly serves as a constructive method for finding an instrument sequence that produces a desired output sequence.

First we introduce some terminology. We will call $l$, the minimal period of time which passes till the target variable $\tilde{y}_{i}(t+l)$ is affected by one of the policy variables $\tilde{u}_{j}(t), j=1, \ldots, m$, the characteristic number $\rho_{i}(t)$ [see Nijmeijer and van der Schaft (1990)]. Formally,

$$
\rho_{i}(t)=\min _{j \in \mathbb{N}}\left\{j \mid C_{i}(t+j) A(k+j ; k+1) B(t) \neq 0\right\} .
$$

with $A(t+s ; t)=A(t+s-1), \ldots, A(t), s \geq 1$, and $A(t, t)=I_{n}$.

With these characteristic numbers $\rho_{1}(t), \ldots, \rho_{m}(t)$ we form the $(m \times m)$ matrix $M(t)$, called the decoupling matrix:

$$
M(t)=\left(\begin{array}{c}
C_{1}\left(t+\rho_{1}(t)\right) A\left(k+\rho_{1}(t) ; k+1\right) B(t) \\
\vdots \\
C_{m}\left(t+\rho_{m}(t)\right) A\left(k+\rho_{m}(t) ; k+1\right) B(t)
\end{array}\right)
$$

Now we give the announced algorithm. We shall do this for the case $m=p=2$. The more general case, $m=p>2$, follows the same lines and will not be given here; see Maas (1991) or Nijmeijer and van der Schaft (1990). 


\section{Algorithm}

Step 1: Define for model (5) with $m=p=2$ the characteristic numbers $\rho_{1, i}(t):=\rho_{i}(t)$ for $i=1,2$ and form the decoupling matrix $M(t)$. Assume that the rank of $M(t)$ is constant for all $t$. If the rank of the decoupling matrix is equal to 2 , we can construct a 'feedback',

$$
\tilde{u}(t)=M(t) \tilde{q}(t)+N(t) \tilde{v}(t)
$$

such that

$$
\tilde{y}_{i}\left(t+\rho_{1, i}\right)=\tilde{v}_{i}(t), \quad i=1,2 .
$$

We are done since we know that model (5) is uniform path controllable because we can construct static feedback (7) which decouples the model [see Nijmeijer (1989)]. If, on the other hand, the rank of $M(t)$ is equal to 1 , we can construct a feedback of the form (7) with $N$ a nonsingular $(2 \times 2)$-matrix such that

$$
\tilde{y}_{1}\left(t+\rho_{1,1}\right)=\tilde{v}_{1}(t)
$$

and $\tilde{y}_{2}\left(t+\rho_{1,2}\right)$ only depends on $\tilde{v}_{1}(t)$ and not on $\tilde{v}_{2}(t)$.

Step 2: We introduce as a dynamic mechanism the following $n$-fold time delay for the input $\tilde{v}_{1}$ :

$$
\begin{aligned}
& \tilde{z}_{1}(t+1)=\tilde{z}_{2}(t), \\
& \tilde{z}_{2}(t+1)=\tilde{z}_{3}(t), \\
& \vdots \\
& \tilde{z}_{n}(t+1)=\tilde{w}(t), \\
& \tilde{v}_{1}(t)=\tilde{z}_{1}(t) .
\end{aligned}
$$

System (5) together with feedback (7) and mechanism (9) form a new system:

$$
\begin{aligned}
& \tilde{q}(t+1)=(A(t)+B(t) M(t)) \tilde{q}(t)+B(t) N(t)\left(\begin{array}{l}
\tilde{z}_{1}(t) \\
\tilde{v}_{2}(t)
\end{array}\right), \\
& \tilde{z}_{1}(t+1)=\tilde{z}_{2}(t), \\
& \tilde{z}_{2}(t+1)=\tilde{z}_{3}(t), \\
& \vdots \\
& \tilde{z}_{n}(t+1)=\tilde{w}(t),
\end{aligned}
$$




$$
\tilde{y}(t)=C(t) \tilde{q}(t)
$$

with instruments $\left(\tilde{w}, \tilde{v}_{2}\right)$, state $\left(\tilde{q}, \tilde{z}_{1}, \ldots, \tilde{z}_{n}\right)$, and targets $\left(\tilde{y}_{1}, \tilde{y}_{2}\right)$.

Define the characteristic numbers $\rho_{2, i}(t):=\rho_{i}(t)$ now for model $(10)$. Form the decoupling matrix, say $M^{2}(t)$, and assume that the rank of $M^{2}(t)$ is constant. In case the rank is equal to 1, model (5) is not uniform path controllable; see Nijmeijer (1990). If the rank is 2, then we can construct a static feedback law depending on the state $\left(\tilde{q}, \tilde{z}_{1}, \ldots, \tilde{z}_{n}\right)$ such that with this feedback applied to $(10)$ it follows that

$$
\tilde{y}_{i}\left(t+\rho_{2, i}\right)=\hat{v}_{i}(t), \quad i=1,2,
$$

with $\hat{v}$ a new instrument variable. As before, this yields that the original system is uniform path controllable.

Remark. The above algorithm does not give positive or negative results on dynamic path controllability in case the ranks of the matrices $M(t)$ and $M^{2}(t)$ depend on $t$. In that - nongeneric - situation a further analysis is required.

Another way for studying (uniform) target path controllability for the linear time-varying system (5) is described in Engwerda (1988a, b). The following result is illustrative:

Theorem 1. Model (5) is uniform path controllable if and only if the rank of the matrix $M(0 ; \alpha, l)$ is equal to $m l$ with $M(0 ; \alpha, l), a(m l \times m(\alpha+l-1))$-matrix, defined as

$$
\begin{gathered}
M(0 ; \alpha, l)= \\
\left(\begin{array}{cccc}
C(\alpha+l-1) B(\alpha+l-2) & C(\alpha+l-1) A(\alpha+l-2) B(\alpha+l-3) & \ldots & \ldots \\
0 & C(\alpha+l-2) B(\alpha+l-3) & & \\
\vdots & \ddots & \ddots & \\
0 & \cdots & 0 & C(\alpha) B(\alpha-1) \\
& & C(\alpha+l-1) A(\alpha+l-1 ; 1) B(0) \\
& & \vdots & \\
& & & \\
& & &
\end{array}\right),(1)
\end{gathered}
$$


with

$$
\begin{aligned}
A(t+s ; t) & =A(t+s-1) \ldots A(t), & & s \geq 1, \\
& =I, & & s=0 .
\end{aligned}
$$

Proof. This theorem is taken from Engwerda $(1988 \mathrm{a}, \mathrm{b})$ in which he proves the connection between $T P C(t ; \alpha, l)$ and a rank condition like (11) for arbitrary initial time $t$. We have taken $t=0$ here.

Remark. In contrast with the foregoing algorithm the construction of an instrument sequence that produces a desired target path is using this theorem not immediate.

\section{Nonlinear path controllability}

Also in the nonlinear case one can use an algorithm like the one mentioned in the previous section to check the local dynamic path controllability of model (4). Therefore we have to generalize the characteristic number and decoupling matrix to the nonlinear situation.

First we define $h_{i}^{0}=h_{i}$, and then calculate the derivative $h_{i}^{0} \circ f$ with respect to $u$ :

$$
\frac{\partial h_{i}^{0}}{\partial u}(f(q, u))=\left(\frac{\partial h_{i}^{0}}{\partial u_{1}}(f(q, u)) \ldots \frac{\partial h_{i}^{0}}{\partial u_{1}}(f(q, u))\right)
$$

The row vector (12) depends in an analytic way on $q$ and $u$. Therefore we have that either this vector is nonzero in an open and dense subspace $O_{i}$ of $\mathscr{Q} \times \mathscr{U}$, or (12) vanishes for all $(q, u) \in \mathscr{Q} \times \mathscr{U}$. In the first case we define the characteristic number $\rho_{i}$ equal to 1 , else we set $h_{i}^{1}(q)=h_{i}^{0}(f(q, u))$ since $h_{i}^{0}(f(q, u))$ does not depend on $u$ if $(12)$ vanishes for all $(q, u)$. We proceed by looking at the next iteration, i.e., the row vector

$$
\frac{\partial h_{i}^{1}}{\partial u}(f(q, u))
$$

We will define $\rho_{i}=2$ if this vector is unequal to zero almost everywhere (in an open and dense subspace $O_{i}$ of $\mathscr{Q} \times \mathscr{U}$ ), otherwise we will continue with $h_{i}^{2}(q)=h_{i}^{1}(f(q, u))$. If none of the vectors is unequal to zero, then we define $\rho_{i}=\infty$. Note that, due to the fact that both $f$ and $h$ are analytic mappings the row vectors are either identical zero or else are nonzero in an open and dense part of the $\mathscr{Q} \times \mathscr{U}$-space. 
Under the condition that all characteristic numbers $\rho_{i}$ are finite, we can define the decoupling matrix

$$
M(q, u)=\left(\begin{array}{c}
\frac{\partial}{\partial u} h_{1}^{\rho_{1}-1}(f(q, u)) \\
\vdots \\
\frac{\partial}{\partial u} h_{m}^{\rho_{m}-1}(f(q, u))
\end{array}\right)
$$

for all $(q, u)$, in the open and dense subspace $O=O_{1} \cap \ldots \cap O_{m}$ of $\mathscr{Q} \times \mathscr{U}$.

Now the algorithm is almost the same as the one given in the previous section, and for that reason will only be given for the case that $m=p=2$. The general case, i.e., arbitrary $m=p$, is given in Nijmeijer (1989).

\section{Algorithm}

Step 1: Define for model (4) the characteristic numbers $\rho_{1, i}:=\rho_{i}$ for $i=1,2$. Form the decoupling matrix $M(q, u)$. Now we will assume that the considered trajectory $(\bar{q}(t), \bar{u}(t))$ lies completely in the open and dense subspace $S \subseteq O$ of $\mathscr{Q} \times \mathscr{U}$ for which the rank of the decoupling matrix is constant. Let

$$
\operatorname{rank} M(q, u)=v_{1}
$$

If $v_{1}$ is equal to 2, then we can construct a local feedback

$$
u(t)=\alpha(q(t), v(t)),
$$

such that

$$
y_{i}\left(t+\rho_{1, i}\right)=v_{i}(t), \quad i=1,2,
$$

as long as the corresponding state remains in $S$. Thus, when $v_{1}=2$, the system is locally path controllable. If $v_{1}=1$, we can construct a local feedback of the form (13) such that

$$
y_{1}\left(t+\rho_{1,1}\right)=v_{1}(t)
$$

and $y_{2}\left(t+\rho_{1,2}\right)$ only depends on $v_{1}(t)$. We now proceed with:

Step 2: We introduce as a mechanism the following $n$-fold time delay for the input $v_{1}$ :

$$
\begin{aligned}
& z_{1}(t+1)=z_{2}(t), \\
& z_{2}(t+1)=z_{3}(t),
\end{aligned}
$$




$$
\begin{aligned}
& z_{n}(t+1)=w(t), \\
& v_{1}(t)=z_{1}(t)
\end{aligned}
$$

System (4) together with feedback (13) and mechanism (14) yields a new system of the form (4) with instruments $\left(w, v_{2}\right)$, state $\left(q, z_{1}, \ldots, z_{n}\right)$, and targets $\left(y_{1}, y_{2}\right)$.

For this new system we again define the characteristic numbers $\rho_{2, i}$. Notice that $\rho_{2,1}=\rho_{1,1}+n$ and $\rho_{2,2} \leq \rho_{1,2}+n$ [see Nijmeijer $\left.(1990 a, b)\right]$. Form the decoupling matrix, and again we assume that the considered trajectory is such that it lies completely in the open and dense subspace $S$ for which the rank of the decoupling matrix is constant $v_{2}$.

If the rank is 1 , then (4) is not locally dynamic path controllable around the considered time path [Nijmeijer $(1990 a, b)]$. Otherwise, if $v_{2}=2$, then we can construct a local feedback such that

$$
y_{i}\left(t+\rho_{2, i}\right)=\hat{v}_{i}(t), \quad i=1,2,
$$

for a new instrument $\hat{v}$, provided we remain in a neighbourhood in $S$. So the system (4) is locally dynamic path controllable around the considered time path.

Remark. The decoupling matrix $M(q, u)$ has constant rank in an open and dense subspace $S$ contained in $O=O_{1} \cap O_{2} \subset \mathscr{Q} \times \mathscr{U}$. In step 1 of the algorithm we impose the condition that the trajectory considered $(\bar{q}(t), \bar{u}(t))$ lies in $S$. Although this is a restrictive condition, it is not very strong since $S$ is an open and dense subspace of $\mathscr{Z} \times \mathscr{U}$.

It follows from, e.g., Nijmeijer and van der Schaft (1990) that in case we can apply the above algorithm - that is, if we work at points in the set $S$ - then we may conclude that around these points we may achieve input-output decoupling via a state feedback (13) locally around these points in $S$ if and only if $v_{1}=2$. As soon as we have a local feedback of the form (13) for the nonlinear system (4) which decouples the system, we can construct a corresponding feedback for the linearized model (5) as will be shown in the following lemma.

Lemma 2. Consider a system of the form (4) and its linearization around a given time path (5). Assume that the considered trajectory of $(4)(\bar{q}(\cdot), \bar{u}(\cdot))$ is contained in $S$ and that

$$
u(t)=\alpha(q(t), v(t))
$$


is a local feedback of (4) which decouples model (4). Let $\bar{v}(t)$ be such that $u(t)=\alpha(q(t), v(t))$, then

$$
\bar{u}(t)=P(t) \tilde{q}(t)+N(t) \tilde{v}(t)
$$

with

$$
P(t)=\frac{\partial \alpha}{\partial q}(\bar{q}(t), \bar{v}(t)), \quad N(t)=\frac{\partial \alpha}{\partial \eta}(\bar{q}(t), \bar{v}(t))
$$

is a feedback of (5) such that (5) with (16) forms a decoupled system.

Proof. System (4) together with feedback (15) leads to the system

$$
\begin{aligned}
q(t+1) & =f(q(t), \alpha(q(t), v(t))), \\
y(t) & =h(q(t)) .
\end{aligned}
$$

The linearization of (17) around the considered trajectory will be

$$
\begin{aligned}
\tilde{q}(t+1) & =(A(t)+B(t) P(t)) \tilde{q}(t)+B(t) N(t) \tilde{v}(t), \\
\tilde{y}(t) & =C(t) \tilde{q}(t) .
\end{aligned}
$$

Now, since (17) is input-output decoupled around the trajectory $(\bar{q}(\cdot), \bar{u}(\cdot))$ in $S$, it will follow by direct computation that the linearization (18) will have the same characteristic numbers and, moreover, by using the chain rule repetitively it also follows that (18) is input-output decoupled.

Using this lemma we can relate the local dynamic path controllability of (4) around a trajectory to the uniform path controllability of the linearization (5) of (4) around that trajectory under the condition that the assumptions made in the above algorithm will hold. This main result is stated in the following theorem:

Theorem 3. Consider a system of the form (4) and its linearization (5) around a given trajectory. Assume that the assumptions made in the algorithm hold, then system (4) will be locally dynamic path controllable around the considered trajectory if and only if linearization (5) is uniform path controllable.

Proof. The proof will be based on the algorithm. It will be given for the case that $m=p=2$. The general case is completely analogous. 
Step 1: Define the characteristic numbers $\rho_{1, i}$ of (4) and $\rho_{1, i}(t)$ of (5). Then calculate the rank of the decoupling matrices $M(q, u)$ and $M(t)$. Because of the assumptions we have made about the fact that the trajectory we consider is completely contained in the open and dense subspace $S$, it follows, using the chain rule, that for all $t$

$$
\operatorname{rank} M(\bar{q}(t), \bar{u}(t))=\operatorname{rank} M(t)=v_{1}
$$

If $v_{1}=2$, we can construct a local feedback which decouples (4) as noted in the algorithm. Using Lemma 2 we can construct a feedback which decouples (4). So model (4) is locally dynamic path controllable and (5) is uniform path controllable.

On the other hand, if $v_{1}=1$, we can construct a local feedback for (4) such that

$$
y_{1}\left(t+\rho_{1,1}\right)=v_{1}(t)
$$

and $y_{2}\left(t+\rho_{1,2}\right)$ only depends on $v_{1}(t)$ provided that we remain in a neighbourhood in $S$. Similarly to the proof of Lemma 2 it is possible to construct a feedback for (5) such that

$$
\tilde{y}_{1}\left(t+\rho_{1,1}(t)\right)=\tilde{v}_{1}(t),
$$

and $\tilde{y}_{2}\left(t+\rho_{1,2}(t)\right)$ only depends on $\bar{v}_{1}(t)$.

Step 2: Introduce the following mechanism for (4):

$$
\begin{aligned}
& z_{1}(t+1)=z_{2}(t), \\
& z_{2}(t+1)=z_{3}(t), \\
& \vdots \\
& z_{n}(t+1)=w(t), \\
& v_{1}(t)=z_{1}(t),
\end{aligned}
$$

and for (5) the following mechanism:

$$
\begin{aligned}
& \tilde{z}_{1}(t+1)=\tilde{z}_{2}(t), \\
& \tilde{z}_{2}(t+1)=\tilde{z}_{3}(t), \\
& \vdots \\
& \tilde{z}_{n}(t+1)=\tilde{w}(t), \\
& \tilde{v}_{1}(t)=\tilde{z}_{1}(t),
\end{aligned}
$$


It is easy to see that model (5) with feedback and mechanism (20) is exactly the linearization of (4) with corresponding fecdback and mechanism (19) around the considered trajectory.

For both systems we will again calculate the characteristic numbers and the decoupling matrices. The rest of the proof will then easily follow in the same lines as step 1 using the algorithms and assumptions.

\section{Examples}

We will explain the theory by two economic examples. The first is a simple growth model of Arrow and Kurz (1970) [see also Aoki(1976)] with one instrument variable and one target variable. The second one is a model of a closed economy [see Wohltmann and Krömer (1984), Nijmeijer (1989), and Nijmeijer and van der Schaft (1990)] in which we define two instruments and two target variables.

\subsection{A two-sector growth model}

Consider the following economic model written in state-space form:

$$
\begin{aligned}
k_{p}(t+1) & =(1-\delta) \mathrm{e}^{-n} k_{p}(t)+s \mathrm{e}^{-n} \gamma\left(k_{p}(t)+k_{g}(t)\right)^{\alpha}, \\
k_{g}(t+1) & =(1-\delta) \mathrm{e}^{-n} k_{g}(t)+\mathrm{e}^{-n} g(t), \\
y(t) & =\gamma\left(k_{p}(t)+k_{g}(t)\right)^{\alpha},
\end{aligned}
$$

where $k_{p}=$ capital of the private sector per capita, $k_{g}=$ capital of the government sector per capita, $y=$ total output per capita, $g=$ government expenditures per capita, and $\delta, s, n, \alpha, \gamma=$ constants.

We assume that $0<\alpha<1$, and first we impose the natural condition that the total capital per capita $\left(k_{p}(t)+k_{g}(t)\right)$ is strictly positive.

We linearize $(21)$ around a trajectory $\left(\bar{g}(t), \bar{k}_{p}(t), \bar{k}_{g}(t), \bar{y}(t)\right)$. Let

$$
\kappa(t)=\left(\bar{k}_{p}(t)+\bar{k}_{g}(t)\right)^{\alpha-1} \text {. }
$$

Then we obtain the following linear time-varying model:

$$
\begin{aligned}
\left(\begin{array}{c}
\tilde{k}_{p}(t+1) \\
\tilde{k}_{g}(t+1)
\end{array}\right) & =A(t)\left(\begin{array}{c}
\tilde{k}_{p}(t) \\
\tilde{k}_{g}(t)
\end{array}\right)+B(t) \tilde{g}(t), \\
\tilde{y}(t) & =C(t)\left(\begin{array}{l}
\tilde{k}_{p}(t) \\
\tilde{k}_{g}(t)
\end{array}\right),
\end{aligned}
$$


where

$$
\begin{aligned}
& A(t)=\left(\begin{array}{cc}
(1-\delta) \mathrm{e}^{-n}+\alpha s \gamma \mathrm{e}^{-n} \kappa(t) & \alpha s \gamma \mathrm{e}^{-n} \kappa(t) \\
0 & (1-\delta) \mathrm{e}^{-n}
\end{array}\right), \\
& B(t)=\left(\begin{array}{c}
0 \\
\mathrm{e}^{-n}
\end{array}\right), \\
& C(t)=(\alpha \gamma \kappa(t) \alpha \gamma \kappa(t)) .
\end{aligned}
$$

It is easily seen that

$$
C(t+1) B(t)=\alpha \gamma \mathrm{e}^{-n} \kappa(k+1) \neq 0,
$$

under the above assumptions. So the matrix condition of Theorem 1 is satisfied, the characteristic number $\rho(t)$ is equal to 1 , and (23) is uniform path controllable.

If we want to steer the output $\tilde{y}(t)$ along a time path $\xi(t), t=1,2, \ldots$, then with the following choice for the instrument $\tilde{g}(t), t=0,1,2, \ldots$,

$$
\tilde{g}(t)=\frac{\xi(t+1)}{\alpha \gamma \mathrm{e}^{-n} \kappa(k+1)}-[(1-\delta)+\alpha s \gamma \kappa(t)]\left(\tilde{k}_{p}(0)+\tilde{k}_{g}(0)\right),
$$

the output $\tilde{y}(t)$ will be equal to the chosen function $\xi(\cdot)$ after a lag of one time step.

Finally, we consider the nonlinear model (21) and calculate its characteristic number $\rho$.

$$
\begin{aligned}
y(t+1)= & \gamma\left\{(1-\delta) \mathrm{e}^{-n}\left(k_{p}(t)+k_{g}(t)\right)\right. \\
& \left.+s \mathrm{e}^{-n} \gamma\left(k_{p}(t)+k_{g}(t)\right)^{\alpha}+\mathrm{e}^{-n} g(t)\right\}^{\alpha} .
\end{aligned}
$$

So $\rho=1$ as long as

$$
g(t) \neq-(1-\delta)\left(k_{p}(t)+k_{g}(t)\right)-s \gamma\left(k_{p}(t)+k_{g}(t)\right)^{\alpha} .
$$

But this is a consequence of the already mentioned condition that the total capital in the economy is assumed to be always strictly positive. Without using the algorithm one can see that (21) is local dynamic path controllable around a given trajectory if for all the points of that trajectory holds that the total capital is greater than zero, which is in agreement with Theorem 3 . In other words, in both (21) and (23) the total output per capita at each time instance 
$t$ can be set at any prescribed value $\xi(t)$ by means of well-chosen government expenditures $g(t)$.

It is useful to note that our assumption that the total capital per capita $\left(k_{p}(t)+k_{g}(t)\right)$ is strictly positive, is essential in our study; see, e.g., the instrument choice in (25). In the perhaps somewhat artificial situation where we drop the assumption that $\left(k_{p}(t)+k_{g}(t)\right)$ is strictly positive - thereby allowing for a negative total capital per capita - the decoupling matrix for the two-sector model (21) is no longer constant for all possible trajectories $\left(\bar{k}_{p}(t), \bar{k}_{g}(t), \bar{g}(t)\right)$, and the same happens for the linearization (23).

In order that the tolal output per capita remains well-defined, we consider the case that $\alpha=\frac{1}{3}$. Following the algorithm of section 4 we find

$$
y(t+1)=\gamma\left(k_{p}(t+1)+k_{g}(t+1)\right)^{1 / 3},
$$

which by substitution of the dynamic equations of (21) enables us to compute the decoupling matrix as

$$
M\left(k_{p}(t), k_{g}(t), g(t)\right)=\frac{1}{3} \gamma \mathrm{e}^{-n} x(t)
$$

where $x(t)=\left(k_{p}(t)+k_{g}(t)\right)^{-2 / 3}$. Clearly the rank of the above decoupling matrix is constant (equal to 1 ) for all points $\left(k_{p}(t), k_{g}(t), g(t)\right)$ for which $x(t) \neq 0$ (notice that this is a less stringent requirement than assuming $x(t)>0$ for all $t$ ). At the same moment we should observe that for linearization (23) the decoupling matrix (24) is identical to (27), and thus has the same singularity. In particular, our algorithmic procedure has resulted in the instrument choice (25), which is not well-defined for all $t$ 's where $x(t)=0$. In a similar way this problem appears for the nonlinear model, and we conclude that our methods cannot adequately handle such singular points and further analysis is required.

\subsection{A model of a closed economy}

As a second example we will look at the following model of a closed economy:

$$
\begin{aligned}
Y(t+1)= & Y(t)+\alpha[C(Y(t))+I(Y(t), R(t), K(t)) \\
& \left.+P^{-1}(t) G(t)-Y(t)\right], \\
R(t+1)= & R(t)+\beta\left[L(Y(t), R(t))-P^{-1}(t) M(t)\right],
\end{aligned}
$$




$$
\begin{aligned}
& K(t+1)=K(t)+I(Y(t), R(t), K(t)), \\
& Y(t)=F(N(t), K(t)), \\
& N(t)=H_{1}(W(t) / P(t)),
\end{aligned}
$$

where $Y=$ real output, $C=$ real private consumption, $I=$ real private netto investment, $R=$ nominal interest rate, $K=$ real capital stock, $P=$ price level, $G=$ nominal government spendings, $L=$ real money demand, $M=$ nominal money stock, $N=$ labour demand, $W=$ nominal wage rate, and $\alpha, \beta=$ strictly positive constants.

Eq. (28) is a dynamic IS cquation and (29) is a dynamic LM equation. The capital accumulation is described via the dynamic Keynesian equation ( 30 ). Eq. (31) is a macroeconomic production function and (32) defines the labour demand as a function of the real wage rate. In fact, this equation follows from an implicit labour demand equation of the form $W(t) / P(t)=f(N(t))$ (with $f=\partial \bar{f} / \partial N$ for some neoclassical production function $\bar{f}$ ), which says that the real wage rate is equal to the marginal product of labour. ${ }^{1}$ In the sequel we will write (32) as

$$
N(t)=H_{1}(W(t) / P(t))=H(W(t), P(t)),
$$

which is the inverse of the marginal product of labour function $f$.

In this model we will take nominal government spending $G$ and nominal money stock $M$ as instruments and real output $Y$ and price level $P$ as target variables. The nominal wage rate $W$ will be assumed to be known and will be handled as exogenous data. As state variables we take real output $Y$, nominal interest rate $R$, and real capital stock $K$.

Assume that $\bar{G}(\cdot), \bar{M}(\cdot), \bar{Y}(\cdot)$, and $\bar{P}(\cdot)$ define a solution of (28)-(33) under certain initial conditions and known data $\bar{W}(\cdot)$. Then we can linearize (28)-(33) around this trajectory. After that we transform the linear model into state-space form. For this transformation we have to assume that, for all $t$,

$$
\begin{aligned}
& \frac{\partial H}{\partial P}(\bar{W}(t), \bar{P}(t)) \neq 0 \\
& \frac{\partial F}{\partial N}(\bar{N}(t), \bar{K}(t))=\frac{\partial F}{\partial N}(H(\bar{W}(t), \bar{P}(t)), \bar{K}(t)) \neq 0
\end{aligned}
$$

\footnotetext{
${ }^{1}$ Under slight modifications the subsequent developments can also be done in case $f$ also depends on $K$, i.e., $f(N(t), K(t))$ and $f=\partial \bar{f} / \partial N$ for a neoclassical function $\bar{f}(N, K)$. Following Wohltmann and Krömer (1984) we assume that $f$ only depends on $N(t)$.
} 
Then the linear time-varying model will be

$$
\begin{aligned}
& \left(\begin{array}{c}
\tilde{Y}(t+1) \\
\tilde{R}(t+1) \\
\tilde{K}(t+1)
\end{array}\right)=A(t)\left(\begin{array}{c}
\tilde{Y}(t) \\
\tilde{R}(t) \\
\tilde{K}(t)
\end{array}\right)+B(t)\left(\begin{array}{c}
\tilde{G}(t) \\
\tilde{M}(t)
\end{array}\right), \\
& \left(\begin{array}{l}
\tilde{Y}(t) \\
\tilde{P}(t)
\end{array}\right)=D(t)\left(\begin{array}{c}
\tilde{Y}(t) \\
\tilde{R}(t) \\
\tilde{K}(t)
\end{array}\right)
\end{aligned}
$$

where

$$
\begin{aligned}
& A(t)= \\
& \left(\begin{array}{ccc}
1+\alpha\left(\frac{\partial C}{\partial Y}(t)+\frac{\partial I}{\partial Y}(t)\right)-\frac{\alpha \bar{P}^{-2}(t) \bar{G}(t)}{\frac{\partial F}{\partial N}(t) \frac{\partial H}{\partial P}(t)} & \alpha \frac{\partial I}{\partial R}(t) & \left.\alpha\left\{\frac{\partial I}{\partial K}(t)+\frac{\bar{P}^{-2}(t) \bar{G}(t) \frac{\partial F}{\partial K}(t)}{\frac{\partial F}{\partial N}(t) \frac{\partial H}{\partial P}(t)}\right\}\right) \\
\beta\left\{\frac{\bar{P}^{-2}(t) \bar{M}(t)}{\frac{\partial F}{\partial N}(t) \frac{\partial H}{\partial P}(t)}+\frac{\partial L}{\partial Y}(t)\right\} & 1+\beta \frac{\partial L}{\partial R}(t) & -\frac{\beta \bar{P}^{-2}(t) \bar{M}(t) \frac{\partial F}{\partial K}(t)}{\frac{\partial F}{\partial N}(t) \frac{\partial H}{\partial P}(t)} \\
\frac{\partial I}{\partial Y}(t) & \frac{\partial I}{\partial R}(t) & 1+\frac{\partial I}{\partial K}(t)
\end{array}\right\}, \\
& B(t)=\left(\begin{array}{cc}
\alpha \bar{P}^{-1}(t) & 0 \\
0 & -\beta \bar{P}^{-1}(t) \\
0 & 0
\end{array}\right), \\
& D(t)=\left(\begin{array}{ccc}
1 & 0 & 0 \\
\frac{1}{\partial F}(t) \frac{\partial H}{\partial P}(t) & 0 & -\frac{\frac{\partial F}{\partial K}(t)}{\frac{\partial F}{\partial N}(t) \frac{\partial H}{\partial P}(t)}
\end{array}\right),
\end{aligned}
$$

in which we set

$$
\frac{\partial C}{\partial Y}(t)=\frac{\partial C}{\partial Y}(\bar{Y}(t)), \quad \frac{\partial I}{\partial Y}(t)=\frac{\partial I}{\partial Y}(\bar{Y}(t), \bar{R}(t), \bar{K}(t)), \quad \text { etc. }
$$


To simplify the notations we shall denote the $(i, j)$ th matrix element of $A$ by $a_{i j}$.

Now we calculate the characteristic numbers of the linearized model (35). It is easy to see that

$$
\rho_{\tilde{Y}}(t)=\rho_{1}(t)=1
$$

by calculation of $\tilde{Y}(t+1)$. By calculation of $\tilde{P}(t+1)$ and using the assumptions (34), we also see that

$$
\rho_{\not{P}}(t)=\rho_{2}(t)=1
$$

The decoupling matrix now equals

$$
M(t)=\left(\begin{array}{cc}
\alpha \bar{P}^{-1}(t) & 0 \\
\frac{\alpha \bar{P}^{-1}(t)}{\partial F}(t+1) \frac{\partial H}{\partial P}(t+1) & 0
\end{array}\right)
$$

so the rank is equal to 1 . We use the following feedback:

$$
\tilde{G}(t)=-\frac{1}{\alpha \bar{P}^{-1}(t)}\left(a_{11}(t), \alpha \frac{\partial I}{\partial R}(t), a_{13}(t)\right)\left(\begin{array}{c}
\tilde{Y}(t) \\
\tilde{R}(t) \\
\tilde{K}(t)
\end{array}\right)+\frac{\tilde{G}^{\prime}(t)}{\alpha \bar{P}^{-1}(t)},
$$

with $\tilde{G}^{\prime}(t)$ denoting a new instrument variable. Then it follows that

$$
\tilde{Y}(t+1)=\tilde{G}^{\prime}(t)
$$

and that $\hat{P}(t+1)$ is independent of $\hat{M}(t)$, but depending on $\tilde{G}^{\prime}(t)$.

Next we introduce a three-fold time delay as a dynamic mechanism:

$$
\begin{aligned}
& \tilde{Z}_{1}(t+1)=\tilde{Z}_{2}(t), \\
& \tilde{Z}_{2}(t+1)=\tilde{Z}_{3}(t), \\
& \tilde{Z}_{3}(t+1)=\tilde{G}^{\prime \prime}(t), \\
& \tilde{G}^{\prime}(t)=\tilde{Z}_{1}(t) .
\end{aligned}
$$


Calculating the characteristic numbers of model (35) with feedback (36) and mechanism (37), we find that

$$
\rho_{2,1}(t)=4, \quad \rho_{2,2}(t)=2
$$

for all $t$, provided that

$$
\frac{\partial F}{\partial K}(t) \neq 0 \quad \text { and } \quad \frac{\partial I}{\partial R}(t) \neq 0 \quad \text { for } t=0,1,2, \ldots
$$

since price level $\tilde{P}$ is influenced by nominal money stock $\tilde{M}$ in two time steps if investments depend on the nominal interest rate and real output depends on the real capital stock.

Assuming that (38) holds we obtain the following decoupling matrix for the new model:

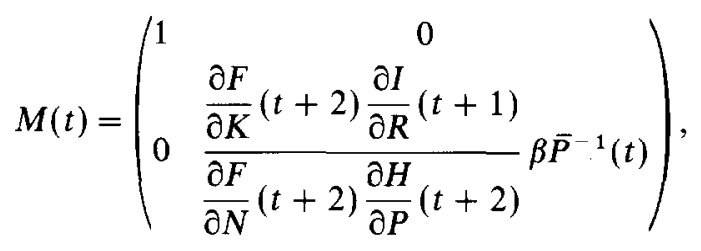

whose rank is equal to 2 under assumptions (38), so there is a feedback depending on the states $\tilde{Y}, \tilde{R}, \widetilde{K}, \tilde{Z}_{1}, \tilde{Z}_{2}, \tilde{Z}_{3}$ and new instruments $\widetilde{G}^{\prime \prime}, \tilde{M}^{\prime}$ such that after applying this feedback to the system (35), (36), (37) we have

$$
\tilde{Y}(t+4)=\tilde{G}^{\prime \prime}(t), \quad \tilde{P}(t+2)=\tilde{M}^{\prime}(t),
$$

and so under the assumptions made model (35) is uniform path controllable.

Now we want to consider the nonlinear model (28)-(33) and try to write it in a state-space form. First consider the equation

$$
N(t)=H(W(t), P(t))
$$

We can transform this into an equation for $P$ as function of $W$ and $N$ by using the Implicit Function Theorem [see Nijmeijer (1989)] when

$$
\frac{\partial H}{\partial P}(\bar{W}(t), \bar{P}(t)) \neq 0,
$$


for all $t$. Assuming (39) we locally can transform the equation into

$$
P(t)=\hat{H}(W(t), N(t)) .
$$

In the same way one can locally transform the equation

$$
Y(t)=F(N(t), K(t))
$$

into an equation for $N$ as function of $Y$ and $K$ by assuming that

$$
\frac{\partial F}{\partial N}(\bar{N}(t), \bar{K}(t)) \neq 0
$$

for all $t$. This yields

$$
N(t)=\hat{F}(Y(t), K(t))
$$

So (28)-(33) [sec Nijmeijer (1989)] in statc-space form will be

$$
\begin{aligned}
& Y(t+1)=f_{1}(Y(t), R(t), K(t), W(t), G(t)), \\
& R(t+1)=f_{2}(Y(t), R(t), K(t), W(t), M(t)), \\
& K(t+1)=f_{3}(Y(t), R(t), K(t)), \\
& Q_{1}(t)=Y(t), \\
& Q_{2}(t)=P(t)=\hat{H}(W(t), \hat{F}(Y(t), K(t))),
\end{aligned}
$$

with $Q_{1}$ and $Q_{2}$ representing the target variables and the functions $f_{1}, f_{2}$, and $f_{3}$ :

$$
\begin{aligned}
& f_{1}(Y(t), R(t), K(t), W(t), G(t))=Y(t)+\alpha[C(Y(t))+I(Y(t), R(t), K(t)) \\
& +(\hat{H}(W(t), \hat{F}(Y(t), K(t))))^{-1} G(t) \\
& -Y(t)] \\
& f_{2}(Y(t), R(t), K(t), W(t), M(t))=R(t)+\beta[L(Y(t), R(t)) \\
& \left.+(\hat{H}(W(t), \hat{F}(Y(t), K(t))))^{-1} M(t)\right], \\
& f_{3}(Y(t), R(t), K(t))=K(t)+I(Y(t), R(t), K(t)) \text {. }
\end{aligned}
$$


First we calculate the characteristic numbers of (43). For $Q_{1}$ we see that

$$
Q_{1}(t+1)=f_{1}(Y(t), R(t), K(t), W(t), G(t))
$$

From the specific structure of $f_{1}$ we conclude that

$$
\frac{\partial f_{1}}{\partial G}(\bar{Y}(t), \bar{R}(t), \bar{K}(t), \bar{W}(t), \bar{G}(t))=(P(t))^{-1} \neq 0
$$

for all $t$, because we assume that the price level of the considered path will always be nonzero.

So $\rho_{1}=1$, and for the second output $Q_{2}$ we see that

$$
\begin{aligned}
Q_{2}(t+1)= & \hat{H}\left(W(t+1), \hat{F}\left(f_{1}(Y(t), R(t), K(t), W(t), G(t)),\right.\right. \\
& \left.\left.f_{3}(Y(t), R(t), K(t))\right)\right)
\end{aligned}
$$

Now $\rho_{2}=1$ if we assume that

$$
\frac{\partial \hat{H}}{\partial N}(\bar{W}(t+1), \bar{N}(k+1)) \frac{\partial \hat{F}}{\partial Y}(\bar{Y}(t+1), \bar{K}(k+1)),
$$

which is equivalent to assumptions (39) and (41).

Now we apply the algorithm. First we introduce a new instrument variable,

$$
G^{\prime}(t)=f_{1}(Y(t), R(t), K(t), W(t), G(t))
$$

Because of (44) we can use the Implicit Function Theorem and locally obtain the equation

$$
G(t)=\hat{f}_{1}\left(Y(t), R(t), K(t), W(t), G^{\prime}(t)\right)^{2} .
$$

In the same way as in the proof of Lemma 2 we can construct a feedback for the linearized model (46) (see also the proof of Theorem 3). This feedback is equivalent to (36). ${ }^{3}$

\footnotetext{
${ }^{2}$ This 'inverse' control law is nothing but a (linear) convex combination of the real output $Y$ and the aggregate demand $D:=C(Y)+I(Y, R, K)+P^{-1} G$ [see Nijmeijer (1989)].

${ }^{3}$ From the previous footnote it is clear that (36) could be interpreted as the linearization of the function $f_{1}$, i.e., of real output $Y$ plus the linearization of aggregate demand $D$.
} 
Now we will apply the following mechanism:

$$
\begin{aligned}
& Z_{1}(t+1)=Z_{2}(t), \\
& Z_{2}(t+1)=Z_{3}(t), \\
& Z_{3}(t+1)=G^{\prime \prime}(t), \\
& G^{\prime}(t)=Z_{1}(t) .
\end{aligned}
$$

The resulting model (43), (46), and (47) will then be

$$
\begin{aligned}
& Y(t+1)=Z(t), \\
& Z_{1}(t+1)=Z_{2}(t), \\
& Z_{2}(t+1)=Z_{3}(t) \\
& Z_{3}(t+1)=G^{\prime \prime}(t), \\
& R(t+1)=f_{2}(Y(t), R(t), K(t), W(t), M(t)), \\
& K(t+1)=f_{3}(Y(t), R(t), K(t)), \\
& Q_{1}(t)=Y(t), \\
& Q_{2}(t)=P(t)=\hat{H}(W(t), \hat{F}(Y(t), K(t))) .
\end{aligned}
$$

Now it is easy to see that $\rho_{2,1}=4$ and that $\rho_{2,2}=2$ by using assumptions (34).

Finally, we introduce the new instrument variable

$$
\begin{aligned}
M^{\prime}(t)= & \hat{H}\left(W(t+2), \hat{F}\left(G^{\prime \prime}(t), f_{3}(Z(t),\right.\right. \\
& \left.\left.\left.f_{2}(Y(t), R(t), K(t), W(t), M(t)), f_{3}(Y(t), R(t), K(t))\right)\right)\right),
\end{aligned}
$$

which we can transform via the Implicit Function Theorem by using the given assumptions to

$$
M(t)=\vartheta\left(W(t+2), G^{\prime \prime}(t), Z(t), Y(t), R(t), K(t), W(t), M^{\prime}(t)\right),
$$

such that

$$
Q_{1}(t+4)=G^{\prime \prime}(t), \quad Q_{2}(t+2)=M^{\prime}(t),
$$

and we conclude that the nonlinear model (28)-(33) is local dynamic path controllable under assumptions (34), (39), (41) and the assumption that the price level will never be equal to zero. It is at this moment unclear what a relevant 
economic interpretation of the equation for the new monetary policy variable $M^{\prime}$ is. One should, however, keep in mind that in any case a law as (50) is in fact only introduced as a means for generating a suitable instrument sequence that produces a prescribed target path.

\section{Concluding remarks}

The ultimate goal of a policy maker is often to steer the target variables in an economy along some desirable time path by manipulating the instrument variables in the economy. In the economic literature this problem has apparently been studied only for linear time-invariant economic models. However, 'since almost all models are also influenced by the outside world' via known exogenous variables, a study of target path controllability for more general economic systems is called for. The present note contributes into this problem in two ways. First, we present an algorithmic procedure for testing whether a linear time-varying model is perfect controllable. Second, we show that under some weak conditions target path controllability of a general nonlinear model is equivalent to that of the corresponding time-varying linearization about a given trajectory. Basically, this result states that for testing the target path controllability the policy maker may restrict himself to work with the linearization of a nonlinear model. Obviously this linearization is much easier to deal with, and the above results indeed justify the common approach of reducing a nonlinear economic model to an appropriate linearization of it. The research described in this paper deals with nonlinear economic models in discrete time. It is our believe that, mutatis mutandis, the results on dynamic path controllability remain valid for systems described in continous time; see Nijmeijer and van der Schaft (1990) for some justification of this claim. Therefore, we may expect that models such as described in Buiter (1979) and Aoki and Canzoneri (1979) may serve as classroom examples for this work in continuous time. What remains for future research is to see if an approximate choice of instruments in the nonlinear model can be taken from the linearized model, thereby using the hypothesis that a linear solution acts as an approximate solution in the nonlinear model. We conclude with the remark that all our investigations strongly depend on the exact knowledge of the economic model, a requirement which is certainly not always met by policy makers in the real world.

\section{References}

Albrecht, F., K.A. Grasse, and N. Wax, 1981, Reproducibility of linear and nonlinear input-output systems, Journal of Mathematical Analysis and Applications 79, 178-202.

Aoki, M., 1974, Non-interacting control of macroeconomic variables: Implications on policy mix considerations, Journal of Economics 2, 261-281.

Aoki, M., 1975, On a generalization of Tinbergen's condition in the theory of policy to dynamic models, Review of Economic Studies 42, 293-296. 
Aoki, M., 1976, Optimal control and system theory in dynamic economic analysis (North-Holland, Amsterdam).

Aoki, M. and M. Canzoneri, 1979, Sufficient conditions for control of target variables and assignment of instruments in dynamic macroeconomic models, International Economic Review 20, 605-615.

Arrow, K. and M. Kurz, 1970, Public investment, the rate of return, and optimal fiscal policy (Johns Hopkins Press, Baltimore, MD).

Brockett, R.W. and M.D. Mesarovic, 1965, The reproducibility of multivariable systems, Journal of Mathematical Analysis and Applications 11, 548-563.

Buiter, W.H., 1979, Unemployment-inflation trade-offs with rational expectations in an open economy, Journal of Economic Dynamics and Control 1, 117-141.

Chow, G.C., 1977, An approach to feedback control of nonlinear econometric systems, in: M.D. Intriligator ed., Frontiers of quantitative economics, Papers invited for presentation at the Econometric Society Third World Congress, Toronto, 1975, 263-279.

Engwerda, J.C., 1988a, Regulation of linear discrete time-varying systems, Ph.D. thesis (University of Eindhoven, Eindhoven).

Engwerda, J.C., 1988b, Control aspects of lincar discrete time-varying systems, International Journal of Control 48, 1631-1658.

Fliess, M., 1986, Esquisses pour une theorie des systemes nonlineaires en temps descret, Rendiconti del Seminario Matematico Università e Politechnico di Torino, 55-67.

Fliess, M., 1991, A fundamental result on the invertibility of discrete time dynamics, in: B. Bonnard, B. Bride, J.P. Gauthier, and I. Kupka, eds., Analysis of controlled dynamical systems, Progress in systems and control theory 8 (Birkhäuser, Boston, MA) 211-223.

Maas, W.C.A., 1991, Regelbaarheid van discrete-tijd niet-lineaire (economische) systemen, Afstudeerverslag (MSc. thesis) (University of Twente, Enschede).

Nijmeijer, H., 1987, Local (dynamic) input-output decoupling of discrete time nonlinear systems, IMA Journal of Mathematical Control and Information 4, 237-250.

Nijmeijer, H., 1989, On dynamic dccoupling and dynamic path controllability in economic systems, Journal of Economic Dynamics and Control 13, 21-39.

Nijmeijer, H., 1990a, Regulation of economic models, Nieuw Archief voor Wiskunde 4, no. 8, $361-373$.

Nijmeijer, H., 1990b, Remarks on the control of discrete time nonlinear systems, in: B. Jakubczyk, K. Malanowski, and W. Respondek, eds., Perspectives in control theory: Proceedings of the Sielpia conference, Poland, 1988 (Birkhaüser, Boston, MA) 261-276.

Nijmeijer, H. and A.J. van der Schaft, 1990. Nonlinear dynamical control systems (Springer Verlag, New York, NY).

Preston, A.J. and A.R. Pagan, 1982, The theory of economic policy (Cambridge University Press, Cambridge).

Respondek, W., 1990, Right and left invertibility of nonlincar control systems, in: II.J. Sussmann, ed., Nonlinear controllability and optimal control (Marcel Dekker, New York, NY) 133-176.

Respondek, W. and H. Nijmeijer, 1988, On local right-invertibility of nonlinear control systems, Control Theory and Advanced Technology 4, 325-348.

Sain, M.K. and J.L. Massey, 1969, Invertibility of linear time-invariant dynamical systems, IEEE Transactions on Automatic Control AC-14, 141-149.

Tinbergen, J., 1952, On the theory of economic policy (North-Holland, Amsterdam).

Wohltmann, H.-W., 1984, A note on Aoki's condition for path controllability of continuous-time dynamic economic systems, Review of Economic Studies 51, 343-349.

Wohltmann, H.-W., 1985a, Function space dependent criteria for target path controllability of dynamical systems, International Journal of Control 41, 709-715.

Wohltmann, H.-W., 1985b, On the controllability of continuous-time macrocconomic models, Journal of Economics 45, 47-66.

Wohltmann, H.-W. and W. Krömer, 1983, A note on Buiter's sufficient condition for perfect controllability of a rational expectations model, Journal of Economic Dynamics and Control 6, 201-205.

Wohltmann, H.-W. and W. Krömer, 1984, Sufficient conditions for dynamic path controllability of economic systems, Journal of Economic Dynamics and Control 7, 315-330.

Wolovich, W.A., 1974, Linear multivariable systems (Springer-Verlag, Berlin). 